\title{
SIMULATION OF SALIX HARVESTING AND PROCESSING TECHNOLOGY USING SOFT CONTAINERS
}

\author{
Sergey P. Karpachev* \\ Bauman Moscow State Technical University (Mytischi branch), 1st Institutskaya street, Mytischi, Moscow region 141005, Russian \\ Federation
}

\begin{abstract}
In recent years, the world agriculture business has developed the technology of growing fastgrowing willow - salix. Salix is a renewable energy resource that is profitable to grow by farmers for their own needs. The economic feasibility of growing salix as a biofuel largely depends on the technology of its harvesting and processing. This article proposes a technological scheme for harvesting and processing salix into fuel wood chips using a tractor-mounted mower-chipper and soft containers (CT), which allows reducing the downtime of the mower-chipper compared to traditional technology using hopper trailer for transporting wood chips (TT). The effectiveness of the CT was evaluated by comparison with the TT. The analysis of efficiency was carried out by results of simulation experiments on mathematical models. As a result of the experiments, it was found that the total time for harvesting and processing salix into chips, including the transportation of chips to the storage site, the CT has no advantages over the TT. The advantages of $\mathrm{CT}$ are in reducing investment costs and minimizing downtime of the mower-chipper.
\end{abstract}

\section{Introduction}

In the structure of the cost of agricultural products, energy costs are up to $30 \%[1,2]$. With rising energy prices, shortage is inevitable.

Renewable energy sources can be an alternative to traditional fuel and energy resources (gasoline, diesel fuel, natural gas, etc.). In recent years, the world agriculture business has developed the technology of growing fast-growing willow - salix, as an energy culture. Salix is grown in Sweden, Denmark, Poland, Hungary, and Czech Republic (fig. 1).

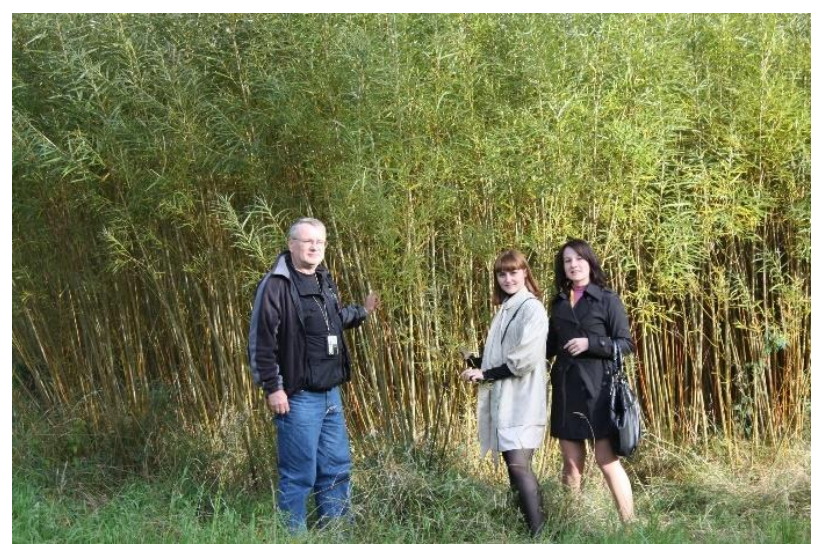

Fig. 1. Salix plantation in Sweden (Source: Author).

Low-productive and unproductive land is suitable for the cultivation of salix. The rapid growth of willows allows to harvest from 9000 to $12000 \mathrm{~kg}$ of dry matter per hectare per year [3].
Despite the obvious advantages of salix as an energy crop, even in Sweden, where the plantations of this crop are the largest and consist of approximately 20000 hectares, the share of fuel chips from salix is less a percent in the total energy balance of the country. The widespread use of sales is hampered by the lack of effective technologies and techniques, especially for its harvesting and processing. Existing technologies are designed for large salix plantations, and equipment for their harvesting and processing costs hundreds of thousands of euro [4]. Such prices can be paid only by large agricultural enterprises that are not always interested in developing their own "green" energy.

The experience of many countries shows that it is profitable to grow energy willow near the processing site and use it for the own needs of the peasant economy: for heating production facilities, greenhouses, drying products, etc. $[5,6,7]$.

\section{Methods}

In this article the technological scheme of harvesting of salix which includes operations of harvesting, processing of salix on fuel chips and its transportation to a storage site is considered. These operations can be performed using a special harvester - a mobile chipper, which works in connection with a transport machine, which has a trailer-hopper for fuel chips. This technology is used in industrial cultivation of salix.

Tractor-mounted mower-chipper can be used for small farms, for example, the equipment of the Danish company Ny Vraa Bioenergi-JF 192-292 [8] (Fig. 2). A

\footnotetext{
${ }^{*}$ Corresponding author: karpachevs@mail.ru
} 
general purpose agricultural tractor can be used as an energy module. In this technology, the tractor must have its own trailer hopper for chips.

It is assumed that after loading the hopper with fuel chips, the harvesting tractor itself transports the chips to a storage site. However, this will increase the overall weight of the machine system, which will inevitably require an increase in tractor engine power. In addition, the productivity of the mower-chipper equipment of the tractor will decrease due to its downtime during the chips transportation phase to the storage site.

In order to reduce the total weight of the machine system, it is proposed to replace the hopper trailer with soft big-bag containers. The container capacity can be 510 tons. The container is installed in place of the hopper and after loading chips dumped on the ground.

Soft containers allow to improve logistics, mechanize loading and unloading operations, increase the efficiency of chips transportation by different modes of transport, etc. [9].

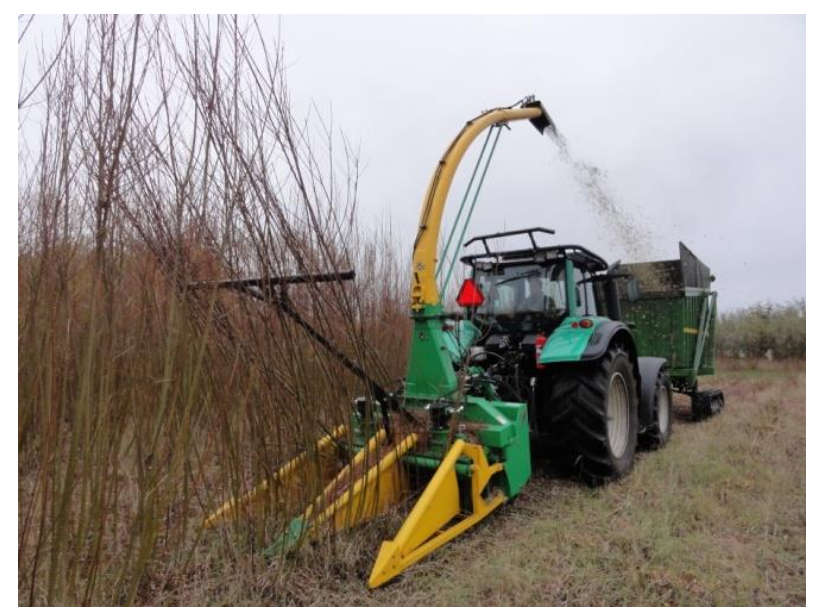

Fig. 2. Agricultural tractor with mower-chipper and hopper trailer for the production of chips from salix (Source: https://nyvraa.dk/energyharvester).

To implement the idea of using soft containers instead of a hopper-trailer, we proposed to provide the tractor with special trailer equipment on which one or more containers are placed for loading [10]. Containers are placed on the platform of the trailer equipment with the ability to tilt them, which allows you to dump the packed containers on the ground by self-dumping.

Container technology allows minimizing the number of specialized machines and equipment, which, hence, minimizes investment costs. The only specialized equipment in the system is a tractor-mounted mowerchipper with a chip pneumatic pipeline. The other machines are general purpose equipment.

On harvesting and processing of salix on fuel wood chips with use of soft containers several variants of technological schemes are possible [11].

In this article the technological scheme with dump of containers on plantations is considered as the simplest (CT) (Fig. 3). The CT includes two phases:

Phase I. Harvesting and processing salix with loading containers with chips and dumping them on the plantation.
Phase II. Collecting containers with chips in the plantation and transporting them to the storage site.

In the first phase, a general-purpose tractor with the mower-chipper (TMC) moves along the technological corridor of the plantation, harvesting salix and processing it into chips. Chips are loaded into a soft container, which is placed on the tractor trailer. After loading the chips, the container is packed, dumped from the trailer and left on the plantation. In place of the unloaded container, an empty container is installed, and the loading cycle is repeated.

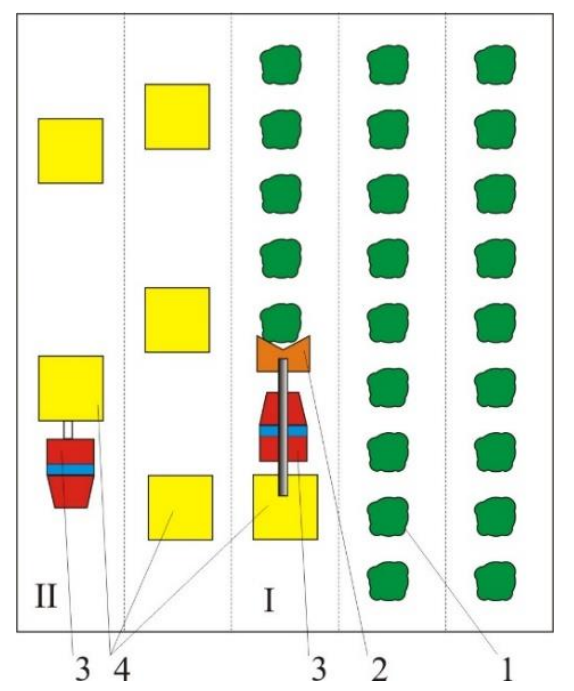

Fig. 3. The operation of the machine system with dumping containers to the plantation: I - phase, II - phase; 1 - salix; 2 mower-chipper; 3 - agricultural tractor; 4 - soft containers (Source: Author).

In the second phase, the same general purpose tractor with a trailer picks up and transports containers with fuel wood chips to the storage site.

Thus, the CT sequentially uses the same generalpurpose tractor at all phases.

Assessing the effectiveness of the CT (Fig. 3) was compared with the technology, which uses a trailer hopper for chips (TT) (Fig. 2). The analysis of efficiency was carried out by results of experiments on mathematical models by methods of computer simulation researches [12].

\section{Results and Discussion}

Simulation experiments were conducted for deferent variable factors. This paper presents simulation results for one variable factor while fixing the others.

The variable factor was the productivity of the TMC at five levels: $1-3-5-7-9$ tons / hour.

The fixed factors were:

- carrying capacity of container (trailer hopper) - 5 tons;

- the size of the plantation - 30 hectares;

- the productivity of the plantation - 10 tons/ha;

- the average distance of chips transportation from the plantation to the storage site $-1000 \mathrm{~m}$.

Some results of the simulation experiments are presented in graphs on fig. 4 and fig. 5 . 


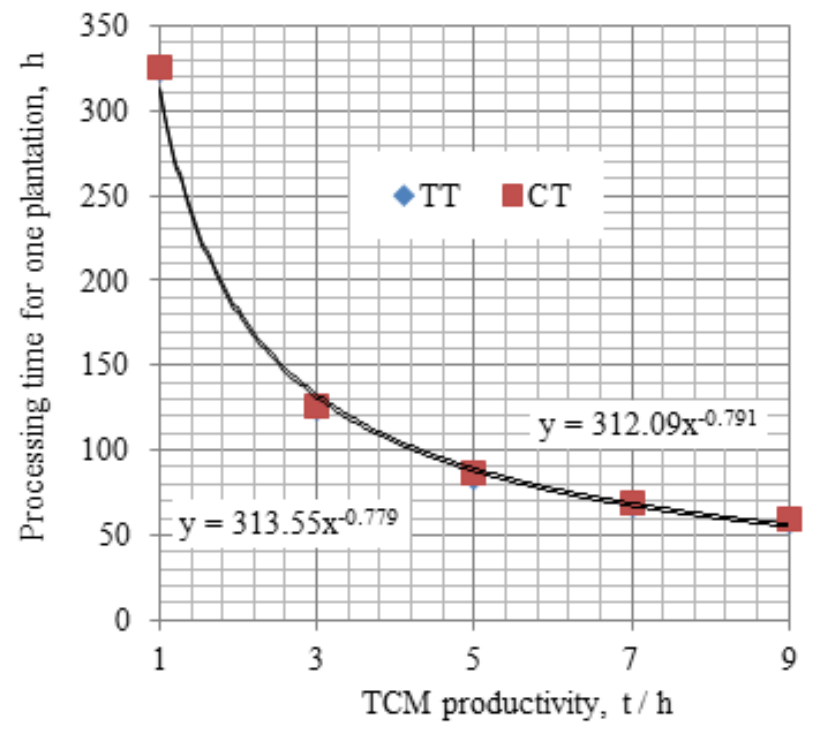

Fig. 4. Dependence of the all processing time, including transportation chips to storage site, for one plantation on the TCM productivity.

As seen in Fig. 4, the processing time for one plantation, including the transportation time, decreases powerfully with increasing of the tractor productivity. It is clearly seen that the total time for processing one plantation by the CT and the TT is almost the same. So, the productivity of the $\mathrm{CT}$ has no advantages over the TT.

The advantages of the $\mathrm{CT}$ are in reducing investment costs and minimizing downtime of the chopping device.

In Fig. 5 shows a graph of dependence of the mowerchipper utilization rate on the productivity of the TMC. From the graph it follows that the utilization rate, expressed as a percentage, decreases with increasing the TMC productivity. At the same time, a significant decrease of the mower-chipper utilization rate is observed only in the TT. So, for example, when the productivity of the TMC is equal to $1 \mathrm{t} / \mathrm{h}$, the mowerchipper utilization rate in the CT is almost $100 \%$, and in the TT - 92.5\%. With an increase in the TCM productivity up to $9 \mathrm{t} / \mathrm{h}$, the mower-chipper utilization rate is reduced. For the TT are from $90 \%$ to $58 \%$, and for the CT - from $99 \%$ to $94 \%$. The decrease in the mowerchipper utilization rate according to the TT occurs because with the increase in the productivity of the TCM, the share of time for transporting chips to the storage site increases. The slight decrease in the mowerchipper utilization rate in the $\mathrm{CT}$ is explained by the change in the share of time for servicing containers.

It should be expected that with the increase in the distance of chips transportation from the plantation to the storage site in the TT, the mower-chipper utilization rate will also decrease. To establish the type of this dependence, we conducted additional experiments. In these experiments, the variable factor was the distance of chips transportation from the plantation to the storage site.

The variable factor was the average distance of chips transportation from the plantation to the storage site: 500 $-1000-1500-2000-2500 \mathrm{~m}$.
The productivity of the TMC was fixed at 9 tons / hour.

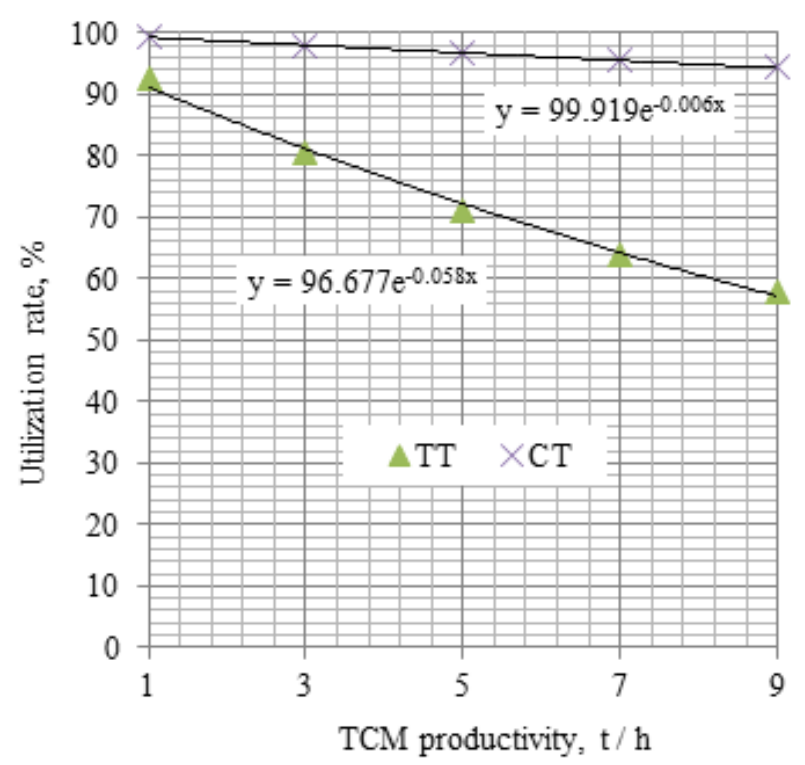

Fig. 5. Dependence of the mower-chipper utilization rate on the TCM productivity.

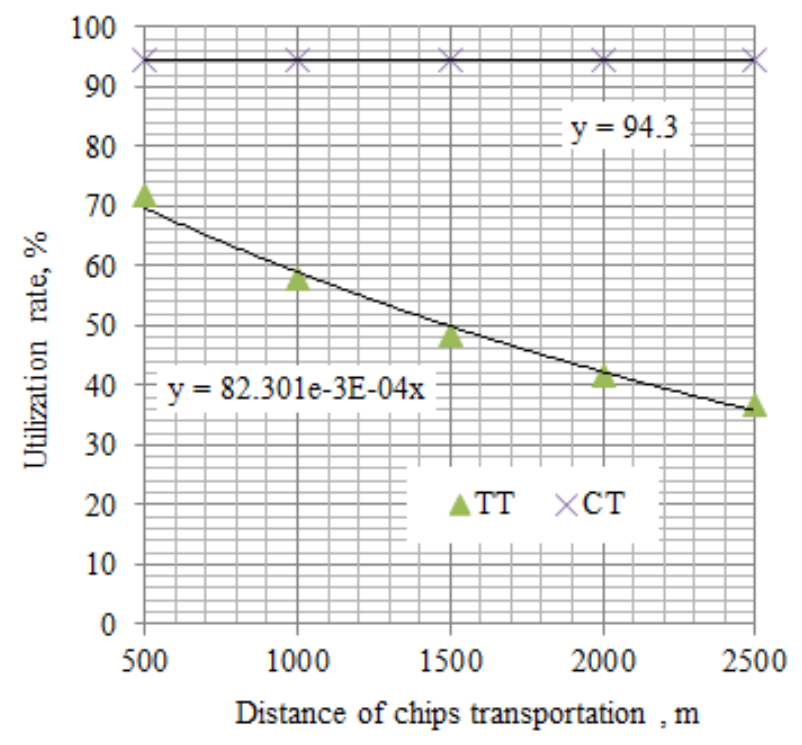

Fig. 6. Dependence of the mower-chipper utilization rate on the distance of chips transportation from the plantation to the storage site.

In Fig. 6 shows a graph of dependence of the mowerchipper utilization rate on the distance of chips transportation from plantation to storage site. From the graph it follows that the utilization rate decreases with increasing the distance of transportation only for the TT.

So, for example, when the distance is equal to $500 \mathrm{~m}$, the mower-chipper utilization rate in the CT is $94.3 \%$, and in the TT $-71.8 \%$. With an increase of distance up to $2500 \mathrm{~m}$, the mower-chipper utilization rate is reduced only for the TT and constant for the CT (94.3\%). For the TT are from $71.8 \%$ to $36.7 \%$. The decrease in the mower-chipper utilization rate for the TT occurs because with the distance increase, the share of time for transporting chips to the storage site increases. 


\section{Conclusions}

1. Proposed a technological scheme for harvesting and processing salix into fuel wood chips using a tractormounted mower-chipper and soft containers (CT), which allows reducing the downtime of the chipper compared to traditional technology using hopper trailer for transporting wood chips (TT).

2. The effectiveness of the CT was evaluated by comparison with the known TT. The analysis of efficiency was carried out by results of simulation experiments on mathematical models.

3. As a result of the experiments, it was found that the total harvest time from the plantation, including the transportation of chips to the storage site, the CT has no advantages over the TT.

4. The advantages of the CT are in reducing investment costs and minimizing downtime of the mower-chipper.

5. The CT can be recommended for cases where a removable mower-chipper is used, which is rented. In case of high productivity of the tractor (more than $5 \mathrm{t} / \mathrm{h}$ ) and long distances of transportation of chips (more than $1000 \mathrm{~m}$ ) it is possible to reduce time of rent of the mower-chipper twice or more.

\section{References}

]1] S.A. Rastimeshin, I.U. Dolgov, D.A. Tikhomirov, M.N. Filkov, The main directions of development of heat and power supply systems for agricultural production, Power supply and energy saving in agriculture, Proceedings of the 8th International Scientific and Technical Conference, 16-17 May 2012, In 5 parts, Part 1, Problems of energy supply and energy saving, M.: GNU VIESH, 384 (2012).

[2] N.M. Morozov, The socio-economic importance of energy conservation in agriculture, Power supply and energy saving in agriculture, Proceedings of the 8th International Scientific and Technical Conference, 16-17 May 2012, In 5 parts, Part 1, Problems of energy supply and energy saving, M.: GNU VIESH, 384 (2012).

[3] R. Pecenka, D. Ehlert, H. Lenz, Efficient harvest lines for Short Rotation Coppices (SRC) in Agriculture and Agroforestry, Agronomy Research, 12, 1, 151-160 (2014) [Electronic resource]. Available at: https://agronomy.emu.ee/vol121/2014_1_17_b5. pdf.

[4] R. Spinelli, C. Nati, N. Magagnotti, G. Picchi, Harvesting poplar medium rotation coppice with light equipment, Proceedings of 23th International Poplar Commission, IPC, FAO, Pechino, China, 168 (2008).

[5] Energy willow (Salix Viminalis) (2019) [Electronic resource]. Available at: http://www.alterenergy.info/eto-interesno/93thebiofuel-technology/781-salix-viminalis).
[6] Energy willow as an option for agribusiness, Case Salix energy, Propositions - The main magazine on agribusiness (2019) [Electronic resource]. Available at: https://propozitsiya.com/energeticheskaya-verbakak-variant-dlyaagrobiznesa-keys-salix-energy.

[7] Is it profitable to grow energy willow in Ukraine? (2019) [Electronic resource]. Available at: https://bio.ukrbio.com/ru/news/15284/.

[8] NyVraaBioenergi (2019) [Electronic resource]. Available at: https://nyvraa.dk/maskiner.

[9] S.P. Karpachev, V.I. Zaprudnov, V.I. Shmyrev, D.V. Shmyrev, A.A. Kamusin, A.K. Redkin, Technological schemes for the development of forest bio-resources for bioenergy using soft containers, Equipment and equipment for the village, 1 (235), 43-46 (2017).

[10] S.P. Karpachev, E.N. Shcherbakov, D.V. Shmyrev, I.P. Karpacheva, K.A. Evstratova, Device to produce chips in the cutting area, The patent for useful model, 140310 (2014).

[11] S.P. Karpachev, E.V. Koverkina, V.I. Shmyrev, Several socio-economic aspects of the production of fuel chips from salix: the case of Russia, Thematic Proceedings named "Ecological Agriculture and Sustainable Development", Research Development Center-FBEE, Belgrade, Serbia, 227-232 (2019) [Electronic resource]. Available at: https:/www.vspep.edu.rs/_img/downsekcija/ 2019/04/ecological_agriculture_and_sustainassu_ development_-_2019.pdf.

[12] S.P. Karpachev, M.A. Bykovskiy, Simulation modelling of the felling-bunching machine logging operation with the multiple-tree accumulating head, 2019 IOP Conf. Ser., Earth Environ. Sci., 226, 012021 (2019). DOI: 10.1088/1755-1315/226/1/012021. 